Research Article

\title{
Numerical Investigation of Chemical Schnakenberg Mathematical Model
}

\author{
Faiz Muhammad Khan, ${ }^{1}$ Amjad Ali, ${ }^{1}$ Nawaf Hamadneh $(1),{ }^{2}$ Abdullah, \\ and Md Nur Alam iD ${ }^{3}$ \\ ${ }^{1}$ Department of Mathematics and Statistics, University of Swat, Pakhtunkhwa, Pakistan \\ ${ }^{2}$ Department of Basic Sciences, College of Science and Theoretical Studies, Saudi Electronic University, Riyadh 11673, Saudi Arabia \\ ${ }^{3}$ Department of Mathematics, Pabna University of Science and Technology, Pabna 6600, Bangladesh
}

Correspondence should be addressed to Nawaf Hamadneh; nhamadneh@seu.edu.sa

and Md Nur Alam; nuralam.pstu23@gmail.com

Received 20 September 2021; Accepted 19 October 2021; Published 9 November 2021

Academic Editor: Taza Gul

Copyright (C) 2021 Faiz Muhammad Khan et al. This is an open access article distributed under the Creative Commons Attribution License, which permits unrestricted use, distribution, and reproduction in any medium, provided the original work is properly cited.

\begin{abstract}
Schnakenberg model is known as one of the influential model used in several biological processes. The proposed model is an autocatalytic reaction in nature that arises in various biological models. In such kind of reactions, the rate of reaction speeds up as the reaction proceeds. It is because when a product itself acts as a catalyst. In fact, model endows fractional derivatives that got great advancement in the investigation of mathematical modeling with memory effect. Therefore, in the present paper, the authors develop a scheme for the solution of fractional order Schnakenberg model. The proposed model describes an auto chemical reaction with possible oscillatory behavior which may have several applications in biological and biochemical processes. In this work, the authors generalized the concept of integer order Schnakenberg model to fractional order Schnakenberg model. We provided the approximate solution for the underlying generalized nonlinear Schnakenberg model in the sense of Caputo differential operator via Laplace Adomian decomposition method (LADM). Furthermore, we established the general scheme for the considered model in the form of infinite series by the aforementioned technique. The consequent results obtained by the proposed technique ensure that LADM is an effective and accurate techniques to handle nonlinear partial differential equations as compared to the other available numerical techniques. Finally, the obtained numerical solution is visualized graphically by MATLAB to describe the dynamics of desired solution.
\end{abstract}

\section{Introduction}

Since the biological processes are not linear systems by nature, which happen at various time scales, therefore, several complex problems arise as a result of fast or slow responds with following interventions or treatment. Thus, to capture an appropriate individual trajectories, the study underconsideration depends on a sequential sample over in appropriate time course. Although various classical methods give a significant insight for the better understanding of a variety of biological processes, but due to some properties like localizing, quantifying and pressibility of measuring revolutionized our thoughts and motivated the researchers and scientists to construct some dynamical methods to tackle various biological phenomena. Most of dynamical and biological phenomena that are involved in the study of chemical theory, fluid dynamics, and mathematical biology have more importance due to explaining the processes related to real life. Such phenomena are usually modulated by linear or nonlinear partial differential equations (PDEs). DEs have ability to predict about the dynamical phenomena around the globe and also used to describe the exponential growth and decay over the time. DEs are having a diverse range of applications in several field, such as physics, engineering, and biology. The researchers use the tool of differential equation, to modulate aforesaid phenomena. Furthermore, 
some useful applications of DEs to modulate the engineering and chemical phenomena can be found in some recent articles (see [1-5]). PDEs often model multidimensional dynamical system, i.e., it can be used to formulate natural phenomena, such as sound, heat, electrostatics, electrodynamics, quantum mechanics, and flow of fluid (see $[6,7])$.

It is important to note that reaction-diffusion systems have been used over decades to study the deep insights of biological systems. More precisely, these models have been used in several biological, physical, environmental, and chemical processes of real life. In reaction-diffusion systems, Brusselator, Lengyel-Epstein, and Schnakenberg models are the most famous due to its applicability and reliable results. These models are used for generating patterns for both biological and chemical systems and so called turning type models. The Schnakenberg model is one of the well-known chemical reaction-diffusion model which was introduced by Schnakenberg in 1979. It is important to note that an auto chemical reaction having oscillatory behavior is precisely described by Schnakenberg model with a verity of the biological and biochemical processes like pattern formations in skin analysis and embryogenesis. Further in biology, it also models the spatial distribution of a morphogen. Science in several biological systems, these types of models involve auto catalytic reactions which natural arises. Therefore, in such reactions, the rate of reaction boost with the reaction proceeds, due to the role of a product acts as catalysts. In tri-molecular reaction, the reaction under consideration plays a role of two species models. Such types of reaction between chemical sources $A$ and $B$ and products $\Phi$ and $\Psi$ are described as:

$$
A \rightleftharpoons \Phi, B \longrightarrow \Psi, 2 \Phi+\Psi \longrightarrow 3 \Phi
$$

where $A$ and $B$ are two chemical sources and $\Phi$ and $\Psi$ are products. A system of reaction-diffusion equations is obtained by using law of mass of action, for the concentrations $\phi(x, t)$ and $\psi(x, t)$ of the products $\Phi$ and $\Psi$ described in (1). The derived nondimensional form of the system $[8,9]$ is given by

$$
\left\{\begin{array}{l}
\frac{\partial \phi(x, t)}{\partial t}=\alpha-\phi(x, t)+\phi^{2}(x, t) \psi(x, t)+D_{1} \frac{\partial^{2} \phi(x, t)}{\partial x^{2}}, \\
\frac{\partial \psi(x, t)}{\partial t}=\beta-\phi^{2}(x, t) \psi(x, t)+D_{2} \frac{\partial^{2} \psi(x, t)}{\partial x^{2}}
\end{array}\right.
$$

where $\phi=\phi(x, t)$ and $\psi=\psi(x, t)$ represent the concentration and $\alpha$ and $\beta$ are positive arbitrary constants and represent the concentration of $A$ and $B . D_{1}$ and $D_{2}$ are the diffusion coefficients of the chemicals $\Phi$ and $\Psi$, for detail study (see [8-11]).

In modern era, the researchers paid keen interest to investigate nonlinear PDEs due to its wide range of applications in physics, engineering, and modern sciences. In last two decades, a considerable number of efforts have been made to investigate field of the fractional order partial differential equations (FOPDEs) in all aspects, such as theoretical, numerical, and applications. These equations provide the hereditary properties and description of memory effect of different phenomena. Fractional order differential operator has the advantage of being a nonlocal operator and possesses greater degree of freedom as compared to conventional differential operator. Fractional calculus has got the consideration of researchers, due to its extensive applications in the aforesaid fields. The mathematical models involving fractional order derivatives are more reliable and great degree of freedom and accuracy as compared to traditional derivatives. In some situation, a mathematical model involving integer order derivative does not describe the real situation. In such circumstances, fractional order derivatives are more reliable to describe these real word problems, (see [12-19]). In this regard, the proposed model has been studied by various researchers from both analytical and numerical points of view (see $[11,20,21]$ ).

After the comprehensive literature review, it was found that mathematical models consist of Caputo fractional order operators that are more accurate and reliable instead of integer order model. Keeping the aforementioned applications of FDEs, the researchers investigated different aspects of various mathematical models. In this continuation, the researchers well explored different aspects of mathematical modeling and published variety of articles (see [2, 22, 23]). Therefore, the researchers investigated different features of aforementioned model. An important class of biochemical model known as Schnakenberg model represents a chemical process, where sudden fluctuation occurs during the reaction. The considered model can be well described by Caputo fractional differential operator instead of integer order derivatives. Therefore, the author used the idea of Caputo fractional order derivatives to generalize the concept of model (2) into Caputo fractional order Schnakenberg model given by

$$
\left\{\begin{array}{l}
\frac{\partial^{\sigma} \phi(x, t)}{\partial t^{\sigma}}=\alpha-\beta \phi(x, t)+\phi^{2}(x, t) \psi(x, t)+D_{1} \frac{\partial^{2 \eta} \phi(x, t)}{\partial x^{2 \eta}}, \\
\frac{\partial^{\sigma} \psi(x, t)}{\partial t^{\sigma}}=\gamma-\phi^{2}(x, t) \psi(x, t)+D_{2} \frac{\partial^{2 \eta} \psi(x, t)}{\partial x^{2 \eta}} \\
0<\sigma, \eta \leq 1
\end{array}\right.
$$

subjected to the initial conditions (ICs)

$$
\begin{gathered}
\phi(x, 0)=h(x), \\
\psi(x, 0)=g(x) .
\end{gathered}
$$

We have established the numerical scheme for aforementioned model with the help of well-known numerical technique called LADM. The proposed technique consists of special polynomial known as Adomian polynomial. The specific class of this polynomial decomposes the nonlinear term involving in the model in the form of series. With the help of Adomian polynomial, the nonlinear term is decomposes as

$$
H(w(x, t))=\sum_{m=0}^{\infty} A_{m}
$$


where $A_{m}$ 's are called Adomian polynomials introduced by Adomian and defined as

$$
A_{m}=\frac{1}{\Gamma(m+1)} \frac{d^{m}}{d \lambda^{m}}\left[H \sum_{i=0}^{m} \lambda^{i} w_{i}(x, t)\right]_{\lambda=0} .
$$

The technique of Laplace Adomian decomposition is the tool to obtain the approximate solution of nonlinear PDEs. LADM is the combination of two powerful techniques, i.e., Adomian decomposition method and Laplace transform. The main advantage of LADM is that it can provide both analytic and numerical solution to a class of nonlinear differential equations (DEs). The considered technique is more superior as compared to the other available techniques, because it gives us particular solutions without finding general solution for DEs. Furthermore, it does not require predefined size declaration like Runge-Kutta method, possess less parameters, and requires no discretization and linearization. In comparison with other analytical techniques, the proposed technique is an efficient and simple tool to investigate numerical solution of nonlinear fractional partial differential equations. The results obtained by this method, ensure the capability and reliability of the proposed method for nonlinear fractional partial differential equations (for detail, see [16, 24, 25]).

In present paper, the authors have generalized the idea of integral order Schnakenberg model to fractional order model in the terms of singular kernal operator. Moreover, we have developed the scheme for the considered fractional model via LADM in the form of infinite series. We have obtained the semianalytical solution for the considered nonlinear model with the help of proposed techniques. The results obtained by the proposed technique ensure that the consider technique is very effective and easy to implement. The numerical simulation is visualized graphically via MATLAB to explain the dynamical behavior of aforementioned model.

\section{Preliminaries}

The concerned section is devoted to the well-known definitions related to fractional calculus and semianalytic techniques, which are helpful in further corresponding in this work.

Definition 1 (see [25]). The LT of a function $g(x, t)$, defined $\forall t \geq 0$, is denoted by $G(x, s)=\mathscr{L}\{g(x, t)\}$ and is given as

$$
G(x, s)=\mathscr{L}\{g(x, t)\}=\int_{0}^{\infty} e^{-s t} g(x, t) d t
$$

where " $\mathscr{L}$ " is called LT operator or Laplace transformation and " $s$ " is the transformed variable.

Definition 2 (see [25]). The noninteger order derivative for the function $\psi$ on the interval $(0, \infty) \times(0, \infty)$ in Caputo sense is defined such as

$$
\begin{aligned}
{ }^{c} \mathscr{D}^{\alpha} \psi(x, t)= & \frac{1}{\Gamma(m-\alpha)} \int_{0}^{t}(t-s)^{m-\alpha-1} \psi^{m}(x, s) d s, \\
& \alpha \in(m-1, m), m \in \mathbb{N},
\end{aligned}
$$

where $m=[\alpha]+1,[\alpha]$ is the integeral part of $\alpha$, and $\alpha$ denotes real number. Now, for $\alpha \longrightarrow m$, the Caputo fractional derivative becomes conventional $n$th order derivative of the function.

Particularly for $\alpha \in(0,1)$,

$$
{ }^{c} \mathscr{D}^{\alpha} \psi(x, t)=\frac{1}{\Gamma(m-1)} \int_{0}^{t} \frac{1}{(t-s)^{\alpha}} \frac{\partial}{\partial s} \psi(x, s) d s .
$$

Definition 3 (see [25]). The LT of Caputo derivatives is given by

$$
\begin{gathered}
\mathscr{L}\left\{{ }^{c} \mathscr{D}^{\alpha} \psi(x, t)\right\}=s^{\alpha} \psi(x, s)-\sum_{k=0}^{m-1} s^{\alpha-k-1} \psi^{k}(x, 0), \\
\alpha \in(m-1, m), m \in N,
\end{gathered}
$$

where $m=[\alpha]+1$ and $[\alpha]$ denote the nonfractional part of $\alpha$.

\section{General Scheme for the Solution Schnakenberg Model}

This section is committed to the general scheme for the solution of fractional order Schnakenberg model via LADM. The fractional order nondimensional Schnakenberg model is given by

$$
\left\{\begin{array}{l}
\frac{\partial^{\sigma} \phi(x, t)}{\partial t^{\sigma}}=\alpha-\phi(x, t)+\phi^{2}(x, t) \psi(x, t)+D_{1} \frac{\partial^{2 \eta} \phi(x, t)}{\partial x^{2 \eta}} \\
\frac{\partial^{\sigma} \psi(x, t)}{\partial t^{\sigma}}=\beta-\phi^{2}(x, t) \psi(x, t)+D_{2} \frac{\partial^{2 \eta} \psi(x, t)}{\partial x^{2 \eta}} \\
0<\sigma, \eta \leq 1
\end{array}\right.
$$

subjected to ICs:

$$
\begin{aligned}
& \phi(x, 0)=h(x), \\
& \psi(x, 0)=g(x),
\end{aligned}
$$

where $\phi=\phi(x, t)$ and $\psi=\psi(x, t)$ represent the concentrations, $\alpha$ and $\beta$ that are positive arbitrary constants and $D_{1}$ and $D_{2}$ are the diffusion coefficients of the substances. 
Applying Laplace transform on (11), we have

$\left\{\begin{array}{l}\mathscr{L}\left\{\frac{\partial^{\sigma} \phi(x, t)}{\partial t^{\sigma}}\right\}=\mathscr{L}\left\{\alpha-\phi(x, t)+\phi^{2}(x, t) \psi(x, t)+D_{1} \frac{\partial^{2 \eta} \phi(x, t)}{\partial x^{2 \eta}}\right\}, \\ \mathscr{L}\left\{\frac{\partial^{\sigma} \psi(x, t)}{\partial t^{\sigma}}\right\}=\mathscr{L}\left\{\beta-\phi^{2}(x, t) \psi(x, t)+D_{2} \frac{\partial^{2 \eta} \psi(x, t)}{\partial x^{2 \eta}}\right\} .\end{array}\right.$

By using properties of Laplace transform, (13) becomes

$$
\left\{\begin{array}{l}
\Phi(x, s)-\frac{1}{s} \phi(x, 0)=\frac{1}{s^{\sigma}} \mathscr{L}\left\{\alpha-\phi(x, t)+\phi^{2}(x, t) \psi(x, t)+D_{1} \frac{\partial^{2 \eta} \phi(x, t)}{\partial x^{2 \eta}}\right\}, \\
\Psi(x, s)-\frac{1}{s} \psi(x, 0)=\frac{1}{s^{\sigma}} \mathscr{L}\left\{\beta-\phi^{2}(x, t) \psi(x, t)+D_{2} \frac{\partial^{2 \eta} \psi(x, t)}{\partial x^{2 \eta}}\right\} .
\end{array}\right.
$$

Now applying inverse Laplace transform on (14) and using ICs, we get

$$
\left\{\begin{array}{l}
\phi(x, t)=h(x)+\mathscr{L}^{-1}\left[\frac{1}{s^{\sigma}} \mathscr{L}\left\{\alpha-\phi(x, t)+\phi^{2}(x, t) \psi(x, t)+D_{1} \frac{\partial^{2 \eta} \phi(x, t)}{\partial x^{2 \eta}}\right\}\right], \\
\psi(x, t)=g(x)+\mathscr{L}^{-1}\left[\frac{1}{s^{\sigma}} \mathscr{L}\left\{\beta-\phi^{2}(x, t) \psi(x, t)+D_{2} \frac{\partial^{2 \eta} \psi(x, t)}{\partial x^{2 \eta}}\right\}\right] .
\end{array}\right.
$$

The nonlinear term $\phi^{2}(x, t) \psi(x, t)$ presenting in (15) is decomposed as

$$
\phi^{2}(x, t) \psi(x, t)=\sum_{n=0}^{\infty} A_{m}
$$

where $A_{m}$ is called Adomian polynomial and defined as

$$
A_{m}=\frac{1}{\Gamma(m+1)} \frac{d^{m}}{d \lambda^{m}}\left[\left(\sum_{i=0}^{m} \lambda^{i} \phi_{i}(x, t)\right)^{2}\left(\sum_{i=0}^{m} \lambda^{i} \psi_{i}(x, t)\right)\right]_{\lambda=0} .
$$

For $m=0$

$$
A_{0}=\phi_{0}^{2}(x, t) \psi_{0}(x, t)
$$

For $m=1$

$$
A_{1}=\phi_{0}^{2}(x, t) \psi_{1}(x, t)+2 \phi_{0}(x, t) \psi_{0}(x, t) \phi_{1}(x, t) .
$$

The assumed solutions $\phi(x, t)$ and $\psi_{i}(x, t)$ are in the form of

$$
\begin{aligned}
& \phi(x, t)=\sum_{i=0}^{\infty} \phi_{i}(x, t), \\
& \psi(x, t)=\sum_{i=0}^{\infty} \psi_{i}(x, t) .
\end{aligned}
$$

Plugging these values in system (15), we have

$$
\left\{\begin{array}{l}
\sum_{i=0}^{\infty} \phi_{i}(x, t)=h(x)+\mathscr{L}^{-1}\left[\frac{1}{s^{\sigma}} \mathscr{L}\left\{\alpha-\sum_{i=0}^{\infty} \phi_{i}(x, t)+\sum_{n=0}^{\infty} A_{n}+D_{1} \frac{\partial^{2 \eta}}{\partial x^{2 \eta}} \sum_{i=0}^{\infty} \phi_{i}(x, t)\right\}\right], \\
\sum_{i=0}^{\infty} \psi_{i}(x, t)=g(x)+\mathscr{L}^{-1}\left[\frac{1}{s^{\sigma}} \mathscr{L}\left\{\beta-\sum_{n=0}^{\infty} A_{n}+D_{2} \frac{\partial^{2 \eta}}{\partial x^{2 \eta}} \sum_{i=0}^{\infty} \psi_{i}(x, t)\right\}\right] .
\end{array}\right.
$$

Comparing both sides of system (21), we have

$$
\begin{aligned}
& \left\{\begin{array}{l}
\phi_{0}(x, t)=h(x), \\
\psi_{0}(x, t)=g(x),
\end{array}\right. \\
& \left\{\begin{array}{l}
\phi_{1}(x, t)=\mathscr{L}^{-1}\left[\frac{1}{s^{\sigma}} \mathscr{L}\left\{\alpha-\phi_{0}(x, t)+A_{0}+D_{1} \frac{\partial^{2 \eta}}{\partial x^{2 \eta}} \phi_{0}(x, t)\right\}\right], \\
\psi_{1}(x, t)=\mathscr{L}^{-1}\left[\frac{1}{s^{\sigma}} \mathscr{L}\left\{\beta-A_{0}+D_{2} \frac{\partial^{2 \eta}}{\partial x^{2 \eta}} \psi_{0}(x, t)\right\}\right],
\end{array}\right. \\
& \left\{\begin{array}{l}
\phi_{n}(x, t)=\mathscr{L}^{-1}\left[\frac{1}{s^{\sigma}} \mathscr{L}\left\{\alpha-\phi_{n-1}(x, t)+A_{n-1}+D_{1} \frac{\partial^{2 \eta}}{\partial x^{2 \eta}} \phi_{n-1}(x, t)\right\}\right], \\
\psi_{n}(x, t)=\mathscr{L}^{-1}\left[\frac{1}{s^{\sigma}} \mathscr{L}\left\{\beta-A_{n-1}+D_{2} \frac{\partial^{2 \eta}}{\partial x^{2 \eta}} \psi_{n-1}(x, t)\right\}\right] .
\end{array}\right.
\end{aligned}
$$

In this manner, we obtain the desired solution given by

$$
\left\{\begin{array}{l}
\phi(x, t)=\sum_{i=0}^{\infty} \phi_{i}(x, t) \\
\psi(x, t)=\sum_{i=0}^{\infty} \psi_{i}(x, t) .
\end{array}\right.
$$

By simple computational work, we get

$$
\begin{gathered}
\left\{\begin{array}{l}
\phi_{0}(x, t)=h(x), \\
\psi_{0}(x, t)=g(x),
\end{array}\right. \\
\left\{\begin{array}{l}
\phi_{1}(x, t)=\left(\alpha-\phi_{0}(x, t)+\phi_{0}^{2}(x, t) \psi_{0}(x, t)+D_{1} \frac{\partial^{2 \eta}}{\partial x^{2 \eta}} \phi_{0}(x, t)\right) \frac{t^{\sigma}}{\Gamma(\sigma+1)}, \\
\psi_{1}(x, t)=\left(\beta-\phi_{0}^{2}(x, t) \psi_{0}(x, t)+D_{2} \frac{\partial^{2 \eta}}{\partial x^{2 \eta}} \psi_{0}(x, t)\right) \frac{t^{\sigma}}{\Gamma(\sigma+1)},
\end{array}\right. \\
\left\{\begin{array}{l}
\phi_{2}(x, t)=\alpha \frac{t^{\sigma}}{\Gamma(\sigma+1)}-\left(\xi-\phi_{0}^{2}(x, t) \zeta-2 \phi_{0}(x, t) \psi_{0}(x, t) \xi-D_{1} \frac{\partial^{2 \eta}}{\partial x^{2 \eta}} \xi\right) \frac{t^{2 \sigma}}{\Gamma(2 \sigma+1)}, \\
\psi_{2}(x, t)=\beta \frac{t^{\sigma}}{\Gamma(\sigma+1)}-\left(\phi_{0}^{2}(x, t) \zeta+2 \phi_{0}(x, t) \psi_{0}(x, t) \xi-D_{2} \frac{\partial^{2 \eta}}{\partial x^{2 \eta}} \zeta\right) \frac{t^{2 \sigma}}{\Gamma(2 \sigma+1)},
\end{array}\right.
\end{gathered}
$$

where

$$
\begin{gathered}
\xi=\alpha-\phi_{0}(x, t)+\phi_{0}^{2}(x, t) \psi_{0}(x, t)+D_{1} \frac{\partial^{2 \eta}}{\partial x^{2 \eta}} \phi_{0}(x, t), \\
\zeta=\beta-\phi_{0}^{2}(x, t) \psi_{0}(x, t)+D_{2} \frac{\partial^{2 \eta}}{\partial x^{2 \eta}} \psi_{0}(x, t) .
\end{gathered}
$$

Thus, the three term solutions are given by 


$$
\left\{\begin{array}{l}
\phi(x, t)=h(x)+\left(2 \alpha-\phi_{0}(x, t)+\phi_{0}^{2}(x, t) \psi_{0}(x, t)+D_{1} \frac{\partial^{2 \eta}}{\partial x^{2 \eta}} \phi_{0}(x, t)\right) \frac{t^{\sigma}}{\Gamma(\sigma+1)}-\left(\xi-\phi_{0}^{2}(x, t) \zeta-2 \phi_{0}(x, t) \psi_{0}(x, t) \xi-D_{1} \frac{\partial^{2 \eta}}{\partial x^{2 \eta}} \xi\right) \frac{t^{2 \sigma}}{\Gamma(2 \sigma+1)}+\cdots \\
\psi(x, t)=g(x)+\left(2 \beta-\phi_{0}^{2}(x, t) \psi_{0}(x, t)+D_{2} \frac{\partial^{2 \eta}}{\partial x^{2 \eta}} \psi_{0}(x, t)\right) \frac{t^{\sigma}}{\Gamma(\sigma+1)}-\left(\phi_{0}^{2}(x, t) \zeta+2 \phi_{0}(x, t) \psi_{0}(x, t) \xi-D_{2} \frac{\partial^{2 \eta}}{\partial x^{2 \eta}} \zeta\right) \frac{t^{2 \sigma}}{\Gamma(2 \sigma+1)}+\cdots
\end{array}\right.
$$

\section{Numerical Discussion}

In this section of research work, we provide some numerical example to illustrate the main work.

Example 1. Let $\alpha=\beta=\eta=1$ and $D_{1}=D_{2}=2$, so the proposed model becomes

$$
\left\{\begin{array}{l}
\frac{\partial^{\sigma} \phi(x, t)}{\partial t^{\sigma}}=1-\phi(x, t)+\phi^{2}(x, t) \psi(x, t)+2 \frac{\partial^{2}}{\partial x^{2}} \phi(x, t) \\
\frac{\partial^{\sigma} \psi(x, t)}{\partial t^{\sigma}}=1-\phi^{2}(x, t) \psi(x, t)+2 \frac{\partial^{2}}{\partial x^{2}} \psi(x, t) \\
0<\sigma, \leq 1
\end{array}\right.
$$

subjected to ICs:

$$
\begin{gathered}
\phi(x, 0)=e^{x} \sin x, \\
\psi(x, 0)=x e^{x} .
\end{gathered}
$$

Applying Laplace transform to system (27), we get

$$
\left\{\begin{array}{l}
\mathscr{L}\left\{\frac{\partial^{\sigma} \phi(x, t)}{\partial t^{\sigma}}\right\}=\mathscr{L}\left\{1-\phi(x, t)+\phi^{2}(x, t) \psi(x, t)+2 \frac{\partial^{2} \phi(x, \mathrm{t})}{\partial x^{2}}\right\}, \\
\mathscr{L}\left\{\frac{\partial^{\sigma} \psi(x, t)}{\partial t^{\sigma}}\right\}=\mathscr{L}\left\{1-\phi^{2}(x, t) \psi(x, t)+2 \frac{\partial^{2} \psi(x, t)}{\partial x^{2}}\right\} .
\end{array}\right.
$$

By using properties of Laplace transform, system (29) becomes

$$
\left\{\begin{array}{l}
\Phi(x, s)-\frac{1}{s} u(x, 0)=\frac{1}{s^{\sigma}} \mathscr{L}\left\{1-\phi(x, t)+\phi^{2}(x, t) \psi(x, t)+2 \frac{\partial^{2} \phi(x, t)}{\partial x^{2}}\right\} \\
\Psi(x, s)-\frac{1}{s} v(x, 0)=\frac{1}{s^{\sigma}} \mathscr{L}\left\{1-\phi^{2}(x, t) \psi(x, t)+2 \frac{\partial^{2} \psi(x, t)}{\partial x^{2}}\right\} .
\end{array}\right.
$$

Now applying inverse Laplace transform on (30) and using ICs, we obtain

$$
\left\{\begin{array}{l}
\phi(x, t)=e^{x} \sin x+\mathscr{L}^{-1}\left[\frac{1}{s^{\sigma}} \mathscr{L}\left\{1-\phi(x, t)+\phi^{2}(x, t) \psi(x, t)+2 \frac{\partial^{2} \phi(x, t)}{\partial x^{2}}\right\}\right], \\
\psi(x, t)=x e^{x}+\mathscr{L}^{-1}\left[\frac{1}{s^{\sigma}} \mathscr{L}\left\{1-\phi^{2}(x, t) \psi(x, t)+2 \frac{\partial^{2} \psi(x, t)}{\partial x^{2}}\right\}\right] .
\end{array}\right.
$$

The nonlinear term $\phi^{2}(x, t) \psi(x, t)$ involved in (31) is decomposed by Adomian polynomial

$$
\phi^{2}(x, t) \psi(x, t)=\sum_{n=0}^{\infty} A_{m} .
$$

Plugging these valves in system (31), we have

$$
\left\{\begin{array}{l}
\sum_{i=0}^{\infty} \phi_{i}(x, t)=e^{x} \sin x+\mathscr{L}^{-1}\left[\frac{1}{s^{\sigma}} \mathscr{L}\left\{1-\sum_{i=0}^{\infty} \phi_{i}(x, t)+\sum_{m=0}^{\infty} A_{m}+2 \frac{\partial^{2}}{\partial x^{2}} \sum_{i=0}^{\infty} \phi_{i}(x, t)\right\}\right], \\
\sum_{i=0}^{\infty} \psi_{i}(x, t)=x e^{x}+\mathscr{L}^{-1}\left[\frac{1}{s^{\sigma}} \mathscr{L}\left\{1-\sum_{m=0}^{\infty} A_{m}+2 \frac{\partial^{2}}{\partial x^{2}} \sum_{i=0}^{\infty} \psi_{i}(x, t)\right\}\right] .
\end{array}\right.
$$

Comparing both sides of system (33), we have

$$
\begin{aligned}
& \left\{\begin{array}{l}
\phi_{0}(x, t)=e^{x} \sin x, \\
\psi_{0}(x, t)=x e^{x},
\end{array}\right. \\
& \left\{\begin{array}{l}
\phi_{1}(x, t)=\mathscr{L}^{-1}\left[\frac{1}{s^{\sigma}} \mathscr{L}\left\{1-\phi_{0}(x, t)+A_{0}+2 \frac{\partial^{2}}{\partial x^{2}} \phi_{0}(x, t)\right\}\right] \\
\psi_{1}(x, t)=\mathscr{L}^{-1}\left[\frac{1}{s^{\sigma}} \mathscr{L}\left\{1-A_{0}+2 \frac{\partial^{2}}{\partial x^{2}} \psi_{0}(x, t)\right\}\right]
\end{array}\right. \\
& \left\{\begin{array}{l}
\phi_{n}(x, t)=\mathscr{L}^{-1}\left[\frac{1}{s^{\sigma}} \mathscr{L}\left\{1-u_{n-1}(x, t)+R_{n-1}+2 \frac{\partial^{2}}{\partial x^{2}} \phi_{n-1}(x, t)\right\}\right], \\
\psi_{n}(x, t)=\mathscr{L}^{-1}\left[\frac{1}{s^{\sigma}} \mathscr{L}\left\{1-R_{n-1}+2 \frac{\partial^{2}}{\partial x^{2}} \psi_{n-1}(x, t)\right\}\right] .
\end{array}\right.
\end{aligned}
$$

Assume the solution in the form of

$$
\left\{\begin{array}{l}
\phi(x, t)=\sum_{i=0}^{\infty} \phi_{i}(x, t) \\
\psi(x, t)=\sum_{i=0}^{\infty} \psi_{i}(x, t) .
\end{array}\right.
$$

By simple computational work, we obtain 

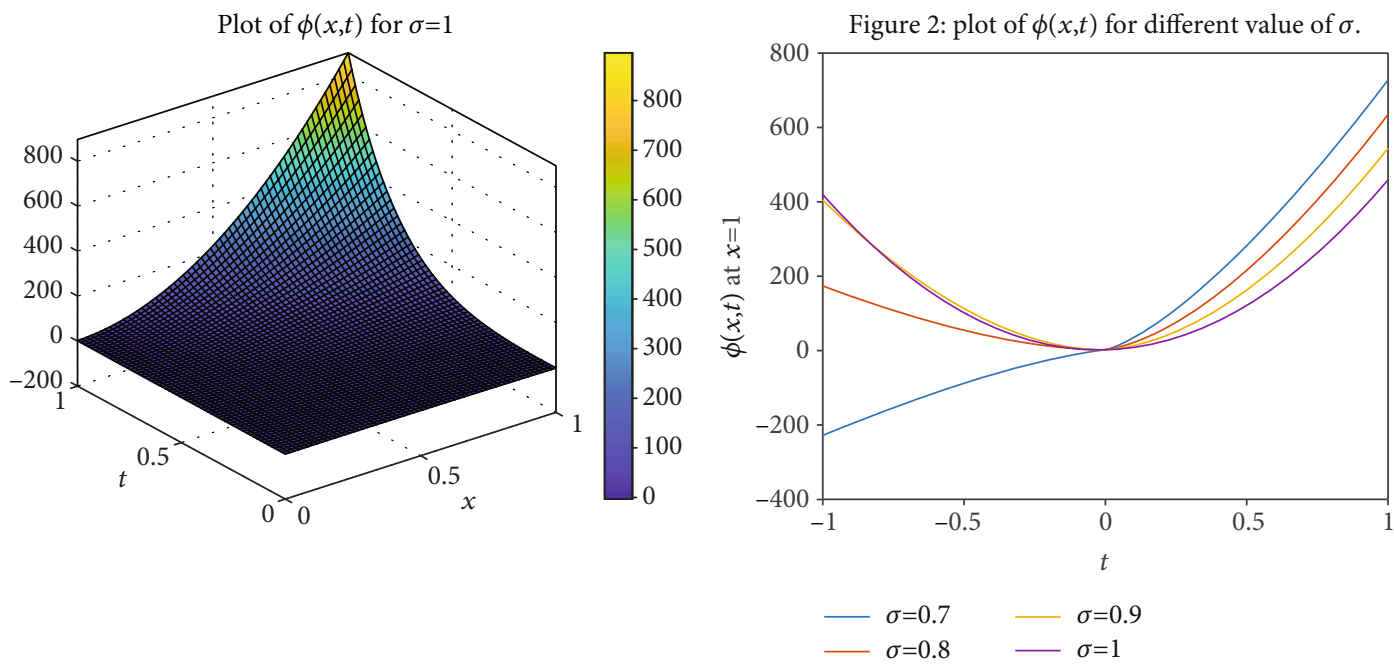

(a)

(b)

Figure 1: $(\mathrm{a}, \mathrm{b})$ Spatial numerical solution of $\psi(x, t)$ in 3D and 2D, respectively.

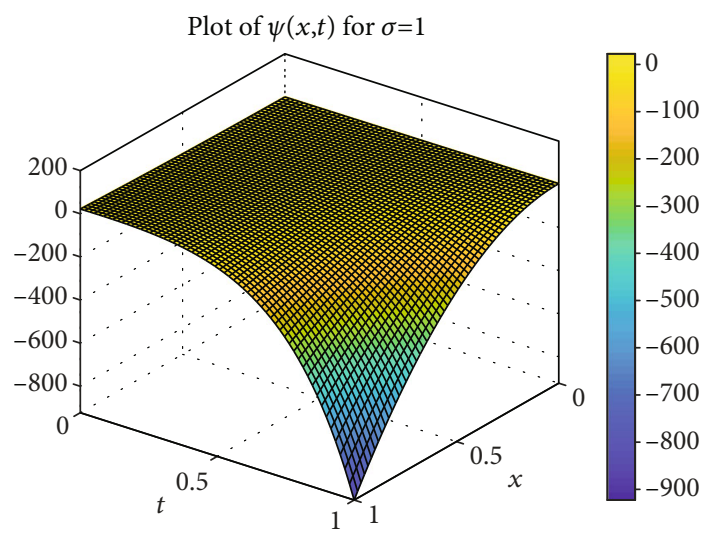

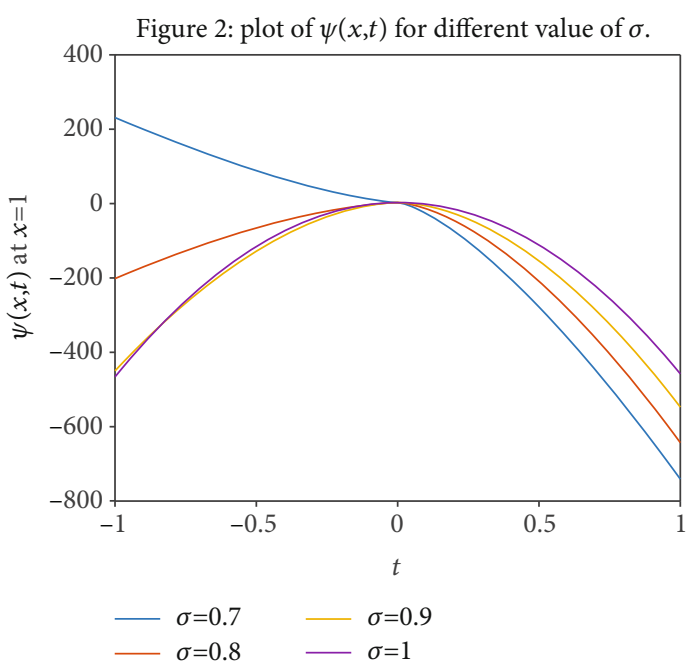

(b)

FIgURe 2: (a, b) Spatial numerical solution of $\phi(x, t)$ in 3D and 2D, respectively.

$$
\left\{\begin{array}{l}
\phi_{1}(x, t)=\left(1-e^{x} \sin x+x e^{3 x} \sin ^{2} x+4 e^{x} \cos x\right) \frac{t^{\sigma}}{\Gamma(\sigma+1)} \\
\psi_{1}(x, t)=\left(1-x e^{3 x} \sin ^{2} x+2 x e^{x}+4 e^{x}\right) \frac{t^{\sigma}}{\Gamma(\sigma+1)}
\end{array}\right.
$$

$\left\{\begin{array}{l}\phi_{2}(x, t)=\frac{t^{\sigma}}{\Gamma(\sigma+1)}+\left(17 x e^{3 x} \sin ^{2} x-1-15 e^{x} \sin x-8 e^{x} \cos x+e^{2 x} \sin ^{2} x-x e^{5 x} \sin ^{4} x+16 e^{3 x} \sin ^{2} x+2 x e^{2 x} \sin x+2 x^{2} e^{5 x} \sin ^{3} x+16 x e^{3 x} \sin 2 x+4 x e^{3 x} \cos 2 x+4 e^{3 x} \sin 2 x\right) \frac{t^{2 \sigma}}{\Gamma(2 \sigma+1)}, \\ \psi_{2}(x, t)=\frac{t^{\sigma}}{\Gamma(\sigma+1)}-\left(e^{2 x} \sin ^{2} x-x e^{5 x} \sin ^{4} x+16 e^{3 x} \sin ^{2} x+2 x e^{2 x} \sin x+2 x^{2} e^{5 x} \sin ^{3} x+4 x e^{3 x} \cos 2 x+16 x e^{3 x} \sin 2 x+4 e^{3 x} \sin 2 x+18 x e^{3 x} \sin x-4 x e^{x}-16 e^{x}\right) \frac{t^{2 \sigma}}{\Gamma(2 \sigma+1)}\end{array}\right.$ 
Continuing the similar fashion, the solution terminated after three terms is given by

$\left\{\begin{array}{l}\left.\phi(x, t)=e^{x} \sin x+\left(2-e^{x} \sin x+x e^{3 x} \sin ^{2} x+4 e^{x} \cos x\right)\right) \frac{t^{\sigma}}{\Gamma(\sigma+1)}+\left(17 x e^{3 x} \sin ^{2} x-1-15 e^{x} \sin x-8 e^{x} \cos x+e^{2 x} \sin ^{2} x-x e^{5 x} \sin ^{4} x+16 e^{3 x} \sin ^{2} x+2 x e^{2 x} \sin x+2 x^{2} e^{5 x} \sin ^{3} x+16 x e^{3 x} \sin 2 x+4 x e^{3 x} \cos 2 x+4 e^{3 x} \sin 2 x\right) \frac{t^{2 \sigma}}{\Gamma(2 \sigma+1)}, \\ \psi(x, t)=x e^{x}+\left(2-x e^{3 x} \sin ^{2} x+2 x e^{x}+4 e^{x}\right) \frac{t^{\sigma}}{\Gamma(\sigma+1)}-\left(e^{2 x} \sin ^{2} x-x e^{5 x} \sin ^{4} x+16 e^{3 x} \sin ^{2} x+2 x e^{2 x} \sin x+2 x^{2} e^{5 x} \sin ^{3} x+4 x e^{3 x} \cos 2 x+16 x e^{3 x} \sin 2 x+4 e^{3 x} \sin 2 x+18 x e^{3 x} \sin x-4 x e^{x}-16 e^{x}\right) \frac{t^{2 \sigma}}{\Gamma(2 \sigma+1)} .\end{array}\right.$

For classical order $\sigma=1$, the solution become

$\left\{\phi(x, t)=e^{x} \sin x+\left(2-e^{x} \sin x+x e^{3 x} \sin ^{2} x+4 e^{x} \cos x\right)\right) t+\left(17 x e^{3 x} \sin ^{2} x-1-15 e^{x} \sin x-8 e^{x} \cos x+e^{2 x} \sin ^{2} x-x e^{5 x} \sin ^{4} x+16 e^{3 x} \sin ^{2} x+2 x e^{2 x} \sin x+2 x^{2} e^{5 x} \sin ^{3} x+16 x e^{3 x} \sin ^{2 x}+4 x e^{3 x} \cos 2 x+4 e^{3 x} \sin ^{2 x}\right) t^{2}$, $\left\{\psi(x, t)=x e^{x}+\left(2-x e^{3 x} \sin ^{2} x+2 x e^{x}+4 e^{x}\right) t-\left(e^{2 x} \sin ^{2} x-x e^{5 x} \sin ^{4} x+16 e^{3 x} \sin ^{2} x+2 x e^{2 x} \sin x+2 x^{2} e^{5 x} \sin ^{3} x+4 x e^{3 x} \cos 2 x+16 x e^{3 x} \sin 2 x+4 e^{3 x} \sin 2 x+18 x e^{3 x} \sin x-4 x e^{x}-16 e^{x}\right) t^{2}\right.$

The Schnakenberg mathematical model actually represents the three steps biochemical processes. In such process, the final product is in the form of $\phi(x, t)$, whose details are given in the introduction of this work. As evident form, the graphical solution of Figures 1(a) and 1(b) shows that the concentration of $\psi(x, t)$ increases and then decreases. While from Figures 2(a) and 2(b), the graph shows that the concentration of $\phi(x, t)$ decreases and then increases. This is a clear evidence that the final product will be obtained in terms of $\phi(x, t)$ with the passage of times which justify the aforementioned three-step process.

\section{Conclusion}

The authors have successfully established the numerical scheme for the generalized fractional order Schnakenberg biochemical model. In order to obtain the desired results, we utilized the tools of well-known numerical technique called Laplace Adomian decomposition method. We have obtained the semianalytic solution for the nonlinear Schnakenberg model in the sense of Caputo differential operator with the help of proposed method. To elaborate our main results, we have provided a numerical example to illustrate our main work. The numerical simulation has been visualized graphically via MATLAB to explain the model's dynamical behavior.

\section{Data Availability}

The data will be available for public after publication.

\section{Conflicts of Interest}

The authors declare that they have no conflicts of interest.

\section{References}

[1] L. Perko, Differential Equations and Dynamical Systems, Springer, New York, NY, USA, 2008.
[2] K. M. Owolabi, "Modelling and simulation of a dynamical system with the Atangana-Baleanu fractional derivative," The European Physical Journal Plus, vol. 133, no. 1, 2018.

[3] K. Alligood, T. Sauer, and C. J. Yorke, An Introduction to Dynamical Systems, Springer-Verlag, New York, NY, USA, 1997.

[4] M. W. Hirsch and S. Smale, Differential Equations, Dynamical Systems, and Linear Algebra, Academic Press, San Diego, CA, USA, 1989.

[5] A. Katok and B. Hasselblatt, Introduction to the Modern Theory of Dynamical Systems, Cambridge University Press, Cambridge, UK, 1995.

[6] E. L. Ince, Ordinary Differential Equations, Dover Public, New York, NY, USA, 1956.

[7] C. Chicone, Ordinary Dfferential Equations with Applications, Springer, New York, NY, USA, 2006.

[8] J. Schnakenberg, "Simple chemical reaction systems with limit cycle behaviour," Journal of Theoretical Biology, vol. 81, no. 3, pp. 389-400, 1979.

[9] Y. Li, "Steady-state solution for a general Schnakenberg model," Nonlinear Analysis: Real World Applications, vol. 12, pp. 1985-1990, 2011.

[10] C. Xu and J. Wei, "Hopf bifurcation analysis in a onedimensional Schnakenberg reaction-diffusion model," Nonlinear Analysis: Real World Applications, vol. 13, no. 4, pp. 1961-1977, 2012.

[11] K. S. Al Noufaey, "Semi-analytical solutions of the Schnakenberg model of a reaction-diffusion cell with feedback," Results in Physics, vol. 9, pp. 609-614, 2018.

[12] S. Saravi and M. Saravi, "A short survey in application of ordinary differential equations on cancer research," American Journal of Computational and Applied Mathematics, vol. 10, pp. 1-5, 2020.

[13] K. L. WANG, K. J. WANG, and C. H. HE, "Physical insight of local fractional calculus and its application to fractional KdV-Burgers-Kuramoto equation," Fractals, vol. 27, no. 7, article 1950122, 2019.

[14] D. Baleanu, A. Fernandez, and A. Akgül, "On a fractional operator combining proportional and classical Differintegrals," Mathematics, vol. 8, no. 3, p. 360, 2020. 
[15] A. Ali, B. Samet, K. Shah, and R. A. Khan, Existence and Stability of Solution to a Toppled System of Differential Equations of Non-Integer Order, Springer, 2017.

[16] A. Ali, K. Shah, and R. A. Khan, "Numerical treatment for traveling wave solutions of fractional Whitham-Broer- Kaup equations," Alexandria Engineering Journal, vol. 57, no. 3, pp. 1991-1998, 2018.

[17] K. S. Miller and B. Ross, An Introduction to the Fractional Calculus and Fractional Differential Equations, A WielyInterscience Publications, John Wiley and Sons, Inc., New York, NY, USA, 1993.

[18] B. Ross, Fractional Calculus and its Applications, Springer Verlag, 1975.

[19] R. Hilfer, Applications of Fractional Calculus in Physics, World Scientific, Singapore, 2000.

[20] M. Ghergu and V. D. Radulescu, Nonlinear PDEs: Mathematical Models in Biology, Chemistry and Population Genetics, Springer, 2012.

[21] M. R. Ricard and S. Mischler, "Turing instabilities at hopf bifurcation," Journal of Nonlinear Science, vol. 19, no. 5, pp. 467-496, 2009.

[22] D. Baleanu, H. Mohammadi, and S. Rezapour, "A fractional differential equation model for the COVID-19 transmission by using the Caputo-Fabrizio derivative," Advances in Difference Equations, vol. 2020, no. 1, 2020.

[23] H. Alrabaiah, A. Ali, F. Haq, and K. Shah, "Existence of fractional order semianalytical results for enzyme kinetics model," Advances in Difference Equations, vol. 2020, no. 1, 2020.

[24] A. Ali, A. Zeb, V. E. Turk, and R. A. Khan, "Numerical solution of fractional order immunology and AIDS via Laplace transform adomian decomposition method," Journal of Fractional Calculus and Applications, vol. 10, no. 1, 2019.

[25] A. Ali, Abdullah, and A. Ahmad, "The solution of Poisson partial differential equations via Double Laplace Transform Method," Partial Differential Equations in Applied Mathematics, vol. 4, p. 100058, 2021. 